in $44 \%$ of cases $(26 / 59)$ no germ was isolated. In one case, the isolated germ was resistant to LNZ. The substitution for fosfomycin by LNZ has led to an estimated extra cost of 2014 euros per month. Conclusions Unavailability of fosfomycin has led to a strong increase in the use of LNZ, particularly for the treatment of NMNBA, causing extra costs and increasing the risk of LNZ resistance. Careful use of this antibiotic, with the contribution of Hospital Pharmacists, should help us preserve its potential.

No conflict of interest.

\section{DGI-073 THE USE OF TRABECTEDIN IN METASTATIC SARCOMA: CASE REPORT OF YOUNG MALE TREATMENT}

\section{doi:10.1136/ejhpharm-2013-000276.339}

G Bellavia, TM Messina Denaro, C Scorsone. Ospedale Giovanni Paolo II, Pharmacy, Sciacca, Italy

Background Trabectedin is a DNA minor groove binder of marine origin. It is indicated for the treatment of adult patients with advanced soft tissue sarcoma after failure of anthracyclines and ifosfamide, or for patients unsuited to receive these agents. In Italy it has been approved since 2009 and it has been included in the Register Monitoring Cancer Drugs.

Purpose To asses the safety and efficacy of treatment for a 28-yearold male patient, with inoperable metastatic sarcoma, not responsive to ifosfamide or anthracyclines

Materials and Methods The oncologist draws up a treatment protocol that is checked by the hospital pharmacist prior to preparation in the Clean Room. The patient was treated with $3 \mathrm{mg}$ of trabectedin in elastomeric pump of $5 \mathrm{ml} / \mathrm{h}$ for 24 hours. This treatment was performed every 21 days.

Results From August 2010 to February 2012 the patient was given trabectedin at the standard dose of $1.5 \mathrm{mg} / \mathrm{m}^{2}$. The first TAC in October 2010 showed stable disease. In March 2011, after 10 cycles, he was still progression-free. The disease started to progress only after 22 cycles. At the beginning of the treatment the patient had abdominal pain, at the end of it, he has neutropenia and increased levels of transaminases. The time to progression (TTP) was 20 months, while in a randomised study TTP was 13.9 months.

Conclusions Trabectedin treatment in soft tissue sarcoma was well tolerated with a good safety profile, demonstrating also a low grade of side effects and a greater time to progression in comparison with the published studies.

No conflict of interest.

\section{DGI-074 TREATMENT AND PROGNOSIS IN PATIENTS WITH WALDENSTROM'S MACORGLOBULINEMIA}

doi:10.1136/ejhpharm-2013-000276.340

A Izquierdo, S Martínez, A Alfaro, M Merchante, L Sanchez-Rubio, A Zorzano, MF Hurtado. San Pedro Hospital, Hospital Pharmacy, Logroño, Spain

Background Waldenstrom's macroglobulinemia (MW) is an uncommon lymphoproliferative disorder of the B cells, associated with overproduction of the monoclonal component Immunoglobulin $\mathrm{M}$ (IgM).

Purpose To analyse the treatment and outcome of patients with MW.

Materials and Methods Observational, retrospective and descriptive study of all patients diagnosed with MW from 2001 to the present day. A cytostatic dispensing programme (OncoGest) and the electronic history (Selene) were used to gather the following data: gender, age, year of diagnosis, previous treatments, treatment regime, adverse reactions. The treatment response was rated according to symptom let-up and decrease in the serum IgM.
Results $8 \mathrm{MW}$ patients were included, their average age was 72 years old (rank: 51-82), of which 50\% were male.

The symptoms with which patients presented before commencing treatment included: asthenia (100\%), anorexia, peripheral neuropathy $(37.5 \%)$, anaemia (25\%), hyperviscosity syndrome $(62.5 \%)$; $40 \%$ of patients required a session of plasmapheresis.

Various treatment regimens were used: Two of the patients commenced treatment with fludarabine, one started with cladribine and two with chlorambucil. Patients with fludarabine had a good response and in the other three cases the response was quite low; as a result, treatment was changed to weekly rituximab until the symptoms stopped and the IgM decreased. Three of the patients started treatment with weekly rituximab with a good response in two of the cases and one had a low response so the treatment was changed to rituximab with cladribine.

All patients except one who is currently receiving rituximab and cladribine have had relapses after the first treatment. They were treated with weekly rituximab until the symptoms stopped, except in two of the cases, who currently continue with maintenance rituximab every two and three months respectively.

As regards tolerance and adverse reactions, neutropenia appeared in just one patient treated with chlorambucil, the treatments were well tolerated by the remaining patients.

Conclusions Various drugs are used for the treatment of MW: chlorambucil, fludarabine, cladribine and rituximab, alone or in combination. The treatment regimen the most commonly used, especially if weekly rituximab, especially for those patients that have had relapses with other treatments. Weekly rituximab is a treatment with a good response rate and is well tolerated.

No conflict of interest.

\section{DGI-075 USE OF BOTULINUM TOXIN TYPE A IN POLAND: SYSTEMATIC REVIEW AND QUESTIONNAIRE SURVEY}

doi:10.1136/ejhpharm-2013-000276.341

' $\underline{\text { S Shergill, }}{ }^{2} \mathrm{~A}$ Miasek, ${ }^{3} \mathrm{M}$ Borowiack, I Czarny-Ozga, ${ }^{3} \mathrm{~W}$ Moczynski, ${ }^{3} \mathrm{~J}$ Stelmachowski, ${ }^{3} \mathrm{~B}$ Slazak, ${ }^{3} \mathrm{M}$ Wachal. 'Allergan Ltd, Health Economics Pricing Reimbursement and Access, Marlow, UK; ${ }^{2}$ Allergan Ltd, Health Economics Pricing Reimbursement and Access, Warsaw, Poland: ${ }^{3}$ Arcana Institute, Health Economics, Krakow, Poland

Background Each botulinum toxin type A product is a unique biological. Due to differences in physicochemical characteristics, measurement of unit doses and dosing regimens they cannot be considered as biosimilars.

Purpose To assess the relative doses used in clinical practise of two different brands of botulinum toxin type A, Dysport and Botox, in focal dystonias (FD), hemifacial spasm (HS) and juvenile cerebral palsy (JCP).

Materials and Methods A systematic review of studies conducted in a variety of countries. The comparison of Dysport with Botox was carried out in accordance with guidelines from the Cochrane collaboration and AHTAPol (Agency for Health Technology Assessment in Poland). Search terms included botulinum toxin type A, dystonic disorders, blepharospasm, hemifacial spasm and cerebral palsy. Concurrently an electronic survey was conducted of eleven Polish doctors, which captured data from 101 of their patients.

Results The systematic review of studies of treating FD and HS with botulinum toxin type A found that where 1.00 unit of Botox is used to treat a patient, between 2.56 and 5.00 units of Dysport are used to treat a patient diagnosed with the same condition. No clinical trials comparing Dysport to Botox were found for JCP. Mean age and percentage of female patients included in the survey was 58.3, 54.7 and 8.9 years; $59.5 \%, 45 \%$ and $40.7 \%$ for FD, HS and JCP respectively. Based on information from patient data collected and surveyed doctors' estimates, the doses for Dysport reflected a broad 
range within and across indications and were on average 4.18-4.78 times those for Botox in FD and HS and 2.41-3.18 times those for Botox in JCP (overall range 2.41-4.78).

Conclusions Botox and Dysport are not interchangeable. The doses used in Poland are consistent with the results of the REAL DOSE study [1]. Treatment is individualised according to patient needs, experience and doctors' preferences.

\section{Reference}

1. Marchetti A et al, Retrospective evaluation of the dose of Dysport and Botox in the clinical management of cervical dystonia or blepharospasm (The REAL DOSE Study), Movement Disorders Vol. 20, No. 8, 2005, pp. 937-944

No conflict of interest.

\section{DGI-076 USE OF COLONY STIMULATING FACTORS (CSF-G) IN FEBRILE NEUTROPENIA IN PATIENTS UNDERGOING CANCER CHEMOTHERAPY}

doi:10.1136/ejhpharm-2013-000276.342

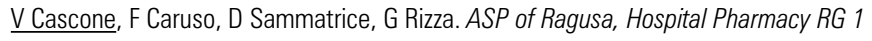
Distrect, Ragusa, Italy

Background The hematopoietic growth factors are a fundamental tool for medical oncologists in the treatment of chemotherapyinduced cytopenia. The proper use of these therapeutic aids plays an important role in terms of reduction of morbidity, mortality and costs.

Purpose To evaluate whether clinical practise follows national guidelines on colony stimulating factors (CSF-G) in the management of hematopoietic toxicity in oncology (AIOM 2010); to investigate the incidence of certain parameters involved in the overall assessment of the risk factors for febrile neutropenia (FN).

Materials and Methods In the first half of 2012, we analysed the CSF-G requirements in patients undergoing cancer chemotherapy. We selected patients treated with cancer chemotherapy, older than 60 years with a risk factor of $\mathrm{FN}>20 \%$, calculated on factors related to the chemotherapy regimen, patient age and type of tumour.

Results We identified 57 patients treated with chemotherapy and CSF-G. Of these, 27 were treated with lenograstim, 24 with pegfilgrastim and 6 with filgrastim. Evaluating the appropriateness of prescribing, according to the parameters identified, showed that only in 12 patients undergoing chemotherapy was a risk factor of FN greater than $20 \%$ observed; of these, 4 were treated with pegfilgrastim, 3 with lenograstim and 5 were not treated ( 3 of which were older than 65 years). We observed that most patients were treated for ovarian, breast or lung cancer or non-Hodgkin's lymphoma, whereas only a small percentage were treated for other cancers such as endometrial, colon, bladder, thymus or biliary tract cancer.

Conclusions The comparison between clinical practise and the AIOM guidelines showed that the use of CSF-G is higher than the level required by the guidelines, when referring exclusively to the 3 major risk factors considered. Therefore, the use of CSF-G in chemotherapy regimens with a low score for febrile neutropenia seems very influenced by additional factors related to the treatment, the patient and the disease.

No conflict of interest.

\section{DGI-077 USE OF EVEROLIMUS IN COMBINATION WITH EXEMESTANE FOR THE TREATMENT OF ADVANCED BREAST CANCER IN A TERTIARY HOSPITAL}

doi:10.1136/ejhpharm-2013-000276.343

M Sanchez, MA ALCACERA, M Gimeno, MA Allende, M Arenere, E Fernández. Hospital Clínico Universitario "Lozano Blesa", Pharmacy, Zaragoza, Spain
Background Everolimus has recently been approved by the European Medicines Agency (EMA) for the treatment of postmenopausal women with advanced breast cancer in combination with exemestane, after treatment failure with letrozole or anastrozole. The approval was based on the results of the BOLERO-2 study.

Purpose To compare the use of everolimus plus exemestane in breast cancer in our hospital with the BOLERO-2 study.

Materials and Methods Retrospective study of all patients treated with everolimus in combination with exemestane from September 2011 to September 2012.

Data source: clinical history and Pharmacy Department records.

The following variables were analysed: age, disease stage, metastases and localization, previous treatment, adverse reactions, duration of treatment, discontinuation of treatment and reasons.

Safety was evaluated by the appearance of adverse reactions.

Results 9 patients with stage IV breast cancer were included. The median age was 54 (range 76-45) years. All patients had bone metastases and 2 had also visceral involvement (pulmonary and hepatic). Previous treatment included: letrozole/anastrozole (7), tamoxifen (6), fulvestrant (5) and chemotherapy (8). 5 patients met the inclusion criteria of the BOLERO-2 study.

The most frequent reason for discontinuation in the BOLERO-2 study was disease progression. In our study 7 patients discontinued, the reasons were: disease progression (3), death (2), and unknown (2). The median duration of treatment was 16 weeks ( 14.6 weeks in the BOLERO-2 study). 2 patients are still continuing with the treatment.

The main side effect was stomatitis (55.6\%) as in the BOLERO-2 study. Other side effects in our study were: epistaxis, rash, fatigue, infection and gastrointestinal reactions.

Conclusions $55.55 \%$ of patients met the inclusion criteria of the BOLERO-2 study and the median duration of treatment was similar.

Stomatitis was the main adverse effect observed.

No conflict of interest.

\section{DGI-078 USE OF SGN35 OR BRENTUXIMAB VEDOTIN IN ANAPLASTIC LARGE-CELL LYMPHOMA: A CASE REPORT IN PAEDIATRICS}

doi:10.1136/ejhpharm-2013-000276.344

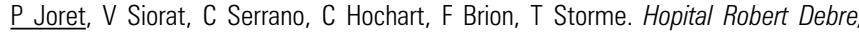
Pharmacy, Paris, France

Background Lymphoma is one of the most frequent haemopathies among children and young adults. Anaplastic large cell lymphoma affects $15 \%$ of such children under 15 years old and $40 \%$ above 15 years old in France. Although the initial treatments are well codified and the efficacy of chemotherapy is well established in most patients, non-responses or relapses with these drugs are leading haemo-oncologists to look for new and effective therapeutic strategies. Thus, SGN35 or brentuximab vedotin is a monoclonal antibody drug conjugate (mADC). It combines an antibody that selectively targets CD30 expression in tumour cells and a cytotoxic drug derived from auristatin. This cell poison is delivered in situ and leads to apoptotic cell death. SGN35 activity is established in Hodgkin's lymphoma and relapsed or refractory systemic anaplastic large-cell lymphomas that are CD30+

Purpose To report the use of brentuximab vedotin in a paediatric case study.

Materials and Methods A literature search was undertaken about the use of brentuximab vedotin in paediatrics. The pharmacy undertook the administrative work to obtain the treatment for the patient.

Results In July 2012, FDA licenced this ADC to treat CD30+ Hodgkin's lymphoma and relapsed or refractory systemic 